\title{
NUEVOS REGISTROS DISTRIBUCIONALES DE NOTOPERLA ENDERLEIN (INSECTA: PLECOPTERA), CON COMENTARIOS TAXONÓMICOS Y BIOLÓGICOS
}

\author{
NEW DISTRIBUTIONAL RECORDS OF NOTOPERLA ENDERLEIN (INSECTA: \\ PLECOPTERA), WITH TAXONOMIC AND BIOLOGICAL COMMENTS
}

Se entregan nuevos registros de distribución para el género Notoperla, sobre la base de la identificación de especímenes imagos. Estos registros permiten extender la distribución geográfica de algunas especies, dejándolas superpuestas con la de otras especies. Por otra parte han permitido tomar observaciones biológicas inéditas de N. magnaspina, que aquí se dan a conocer.

El género Notoperla Enderlein, 1909 es exclusivo del extremo austral de Sudmérica, se encuentra asociado a la Cordillera de los Andes entre los $33^{\circ}$ y $54^{\circ}$ de Latitud Sur, en ambientes de esteros bien oxigenados que frecuentemente se cubren con nieve o pueden interrumpirse durante el invierno.

Illies (1958) propone la familia Abranchioperlidae sobre la base de una ninfa próxima a Gripopterygidae pero desprovista de branquias, posteriormente ésta es integrada a Gripopterygidae como un sinónimo de Notoperla en la subfamilia Leptoperlinae (Illies 1963), con ello la pérdida de las branquias y su reemplazo por respiración cuticular es considera como una apomorfía del género. Illies
(1963) en su revisión de los Gripopterygidae reconoce solo tres especies que se sustituyen latitudinalmente de norte a sur: $N$. archiplatae Illies, 1985, N. tunelina (Navás, 1971) y N. fuegiana (Enderlein, 1905), posteriormente McLellan et al. (2005) adiciona N. macdowalli McLellan \& Mercado, 2005 para Chile y McLellan et al. (2006) a N. fasciata McLellan, 2006 y N. magnaspina McLellan, 2006 ambas exclusivas de Argentina. Froehlich (2010) en su catálogo reconoce siete especies de Notoperla, dado que considera válida a N. conspicua Froehlich 1960 por la recomendación de Zwick (1973).

El escenario taxonómico del género ha sido confuso desde su origen por diversos motivos: 1.para su estudio se ha contado con escasos imagos, en oposición a la abundancia de las ninfas, las que se presentan casi de forma continua a lo largo de la distribución del género. 2.- el género incluye una gran variedad de fenotipos con reducción de las alas, lo que impide el uso de los caracteres asociados a ellas, siendo las hembras macrópteras y los machos micrópteros en $N$. conspicua, $N$. fuegiana, $N$. fasciata y $N$. magnaspina en esta última los machos

1 Departamento de Biología. Facultad de Ciencias Básicas. Universidad Metropolitana de Ciencias de la Educación. José Pedro Alessandri 774. Santiago, Chile; alveras2@gmail.com 
pueden llegar a ser braquípteros (en su descripción original), N. macdowalli es áptera, mientras que en $N$. archiplatae los machos varían desde ápteros hasta macrópteros. Finalmente, en $N$. tunelina las hembras son braquípteras o macrópteras y los machos son macrópteros.

En este contexto la identificación específica puede lograrse casi exclusivamente mediante el estudio genital de imagos macho. Por otra parte, la descripción de nuevas especies posteriores al trabajo de Illies (1963) obliga a replantear las distribuciones de las especies previamente conocidas, en especial aquellas atribuidas a la identificación de ninfas.

En la siguiente lista se muestra la distribución conocida y las nuevas localidades confirmadas por la presencia de imagos:

\section{1.- N. archiplatae (Illies 1958)}

CHILE: Prov. Cordillera, Santiago; Prov. Colchagua; Prov. Nuble, Las Trancas; Prov. Aisén; Prov. Magallanes.

ARGENTINA: Prov. Río Negro; Prov. Tierra del Fuego.

\section{2.- N. conspicua Froehlich 1960}

ARGENTINA: Prov. Tierra del Fuego, Ushuaia.

3.- N. fasciata McLellan 2006

ARGENTINA: Prov. Chubut, Estero la Hoya.

Nuevo: ARGENTINA: Prov. Santa Cruz, Lago Buenos Aires, Camino a los Antiguos. (15,6 Km N Paso Roballos 19-enero-2007 col G.Cheli 4703'27.0"S7149'08.4”W 1329ms$\mathrm{nm})$. Deposito colección IADIZA-Mendoza Argentina.

\section{4.- N. fuegiana (Enderlein 1905)} ARGENTINA: Prov. Tierra del Fuego, Ushuaia.

5.- N. macdowalli McLellan \& Mercado 2005 CHILE: Prov. Cordillera, Santuario Natural Yerba Loca.

6.- N. magnaspina McLellan 2006

ARGENTINA: Prov. Neuquén, Lago Huechulafquen; Prov. Río Negro, Bariloche; Prov. Santa Cruz, Arrollo del Puesto; Prov. Chubut, Río Blanco, Estero la Hoya.
Nuevo: CHILE: Prov. Talca, Estero Azufre; Prov. Coyhaique, Coyhaique Alto Estancia Baño Nuevo. (01-nov.-2008 col. A. Vera). Deposito colección entomológica MNHNSantiago de Chile.

\section{7.- N. tunelina (Navás 1971)}

CHILE: Prov. Ultima Esperanza; Prov. Aysén, Puyuhuapi, Río Murta, Lago General Carrera. ARGENTINA: Prov. Sta. Cruz, Valle Tunél. Nuevo: CHILE: Prov. Aysén; Coyhaique Lago Elizalde; Prov. Ultima Esperanza, Glacial Tyndall (sobre hielo). (17-ene-2007 col. A. Zúñiga). Deposito colección entomológica MNHN-Santiago de Chile.

N. magnaspina es identificada por primera vez para Chile en las Provincias de Talca y Coyhaique, con lo que se extiende la distribución en su límite norte, y la intercalan con la distribución de $N$. tunelina y N. archiplateae. El registro de N. fasciata para la provincia de Santa Cruz, es la segunda localidad donde se identifica esta especie y se ubica solo a $16 \mathrm{~km}$ de la frontera con Chile, superponiendo su distribución con $N$. tunelina. En varias colecciones se encuentran ninfas de Notoperla colectadas en el territorio chileno de Tierra del Fuego, estas podrían haberse atribuido a $N$. fuegiana, sin embargo al reconocerse como válida $N$. conspicua, sería inadecuado identificarlas en una u otra por el momento. La irrupción de estas especies implica que la identificación de las ninfas debe tomarse con precaución ya que la diagnosis de estos estados aún es parcial.

\section{Observaciones biológicas}

La población de Baño Nuevo (Coyhaique Alto) para N. magnaspina, muestra un hábitat (Fig. 1) semejante al señalado para otras especies del género, al voltear los bolones fue posible colectar sumergidos en el agua: ninfas (Fig. 3), exuvias de emergencia e imagos. Los imagos recién emergidos (Fig. 2) permanecen sumergidos, 16 imagos macho fueron colectados en estas condiciones. La emergencia de imagos bajo el agua, nunca antes había sido registrada para Plecoptera, por el contrario existen varias especies donde las ninfas adquieren hábitos anfibios o terrestres antes de mudar a imagos. Puede proponerse a modo de hipótesis que la 

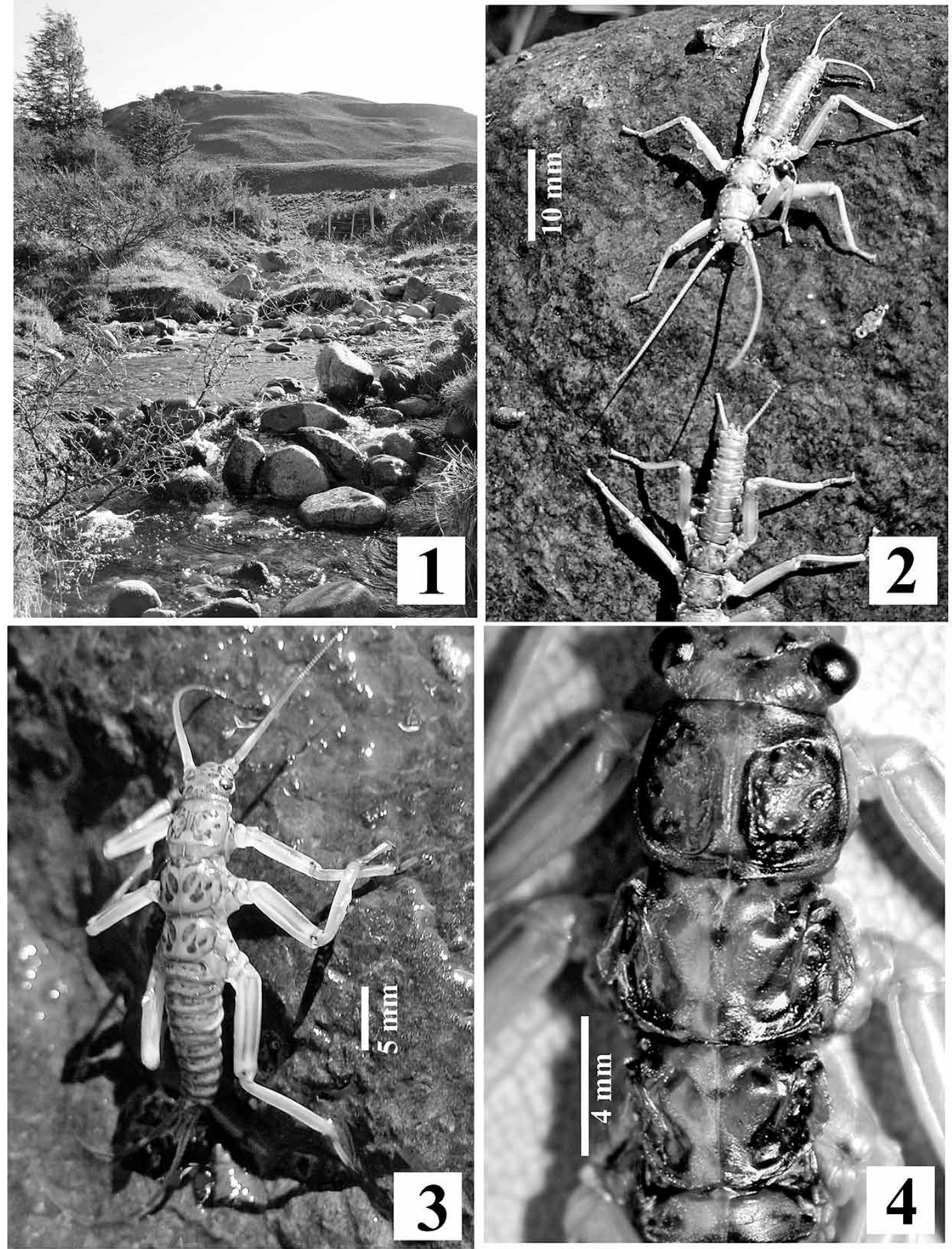

Figs.1-4 N. magnaspina: 1) Estero con bolones en la localidad de Baño Nuevo; 2) Imagos recién emergidos con gotas de agua, en roca recién volteada; 3) Ninfa de último estadio, en roca recién volteada; 4) Tórax de un imago macho con alas micrópteras. 
conducta de los imagos de permanencia en el agua les permita evitar la deshidratación, la depredación o ser arrastrados en las aguas torrentosas donde se desarrollan. De cualquier forma esta conducta se ve favorecida en esta población ya que los imagos son micrópteros (Fig. 4) y no requieren extender sus alas, a diferencia de las poblaciones estudiadas en la descripción original, donde los machos son braquípteros. Además el mecanismo de respiración cuticular de las ninfas podría operar igualmente en los primeros momentos de la vida imaginal. En crianzas de laboratorio, se observa que otros Gripopterygidae de fenotipo macrópteros o braquípteros, emergen siempre en ambiente seco y la extensión de las alas es progresiva y puede tarda varios minutos.

\section{AGRADECIMIENTOS}

Se agradece a quienes me han facilitado especímenes: Rodrigo Pardo y Álvaro Zúñiga. A Federico Ocampo por el préstamo de material desde la Colección IADIZA, a José Mondaca por el traslado del mismo, a Susana Toledo por su ayuda en el trabajo de campo y a los evaluadores del manuscrito que han contribuido a mejorarlo. A los proyectos que han financiado parcialmente este estudio: MECESUP UMC-0204, CONICYT AT-23070183.

\section{LITERATURA CITADA}

Froehlich, C.G. 1960. Some gripopterygids and notonemourines (Plecoptera) from South America. Lunds Universitets Årsskrift 56:3-24

Froehlich, C.G. 2010. Cataloge of Neotropical Plecoptera. Illiesia, 6(12):118-205

Illies, J. 1963. Revision der südamerikanischen Gripopterygidae (Plecoptera). Mitteilungen der Schweizerischen Entomologischen Gesellschaft 36:145-248

McLellan I., M. Mercado \& S. Elliott 2005. A new species of Notoperla (Plecoptera: Gripopterygidae) from Chile. Illiesia 1(5):1-7

McLellan, I., M.L. Miserendino \& M.E.T. Hollmann 2006. Two new species of Notoperla (Plecoptera: Gripopterygidae) and a redescription of Notoperlopsis femina Illies. Zootaxa 1140:53-68

Zwick, P. 1973. Die Plecopteren-Arten Enderleins (Insecta); Revision der Typen. Annales Zoologici 30(16):471-507 